\title{
Improvement of weighted essentially non-oscillatory schemes near discontinuities
}

\author{
Yiqing Shen ${ }^{\mathrm{a}}$, Gecheng Zha ${ }^{\mathrm{b}, *}$ \\ ${ }^{a}$ LHD, Institute of Mechanics, Chinese Academy of Sciences, Beijing 100190, China \\ ${ }^{\mathrm{b}}$ Dept. of Mechanical and Aerospace Engineering, Miami Wind ${ }^{\mathrm{TM}}$ University of Miami, Coral Gables, FL 33124, United States
}

\section{A R T I C L E I N F O}

\section{Article history:}

Received 26 September 2011

Received in revised form 26 November 2013

Accepted 12 February 2014

Available online 21 February 2014

\section{Keywords:}

Shock capturing

WENO scheme

High order scheme

\begin{abstract}
A B S T R A C T
In this article, we analyze the fifth-order weighted essentially non-oscillatory (WENO-5) scheme and show that, at a transition point from smooth region to a discontinuity point or vice versa, the accuracy order of WENO-5 is decreased to third order. A new method is proposed to overcome this drawback by introducing fourth-order fluxes combined with high order smoothness indicator. Numerical examples show that the new method is more accurate near discontinuities with accuracy improved to fourth order.
\end{abstract} (c) 2014 Elsevier Ltd. All rights reserved.

\section{Introduction}

The WENO scheme concept was first proposed by Liu et al. [1] and then improved by Jiang and Shu [2]. WENO schemes are based on ENO (essentially non-oscillatory) schemes [3,4], but use a convex combination of all candidate stencils instead of the smoothest one as in the ENO schemes. The WENO schemes achieve high order accuracy in smooth regions with more compact stencil and have better convergence due to the smoother numerical flux used.

Jiang and Shu [2] analyze and modify the fifth order WENO scheme proposed by Liu et al. [1] and suggest a new way of measuring the smoothness of a numerical solution. Thus a WENO scheme with the optimal $(2 r-1)$ th order accuracy rather than $(r+1)$ th order is obtained. Henrick et al. [5] point out that the original smoothness indicators of Jiang and Shu fail to improving the accuracy order of WENO scheme at critical points, where the first derivatives are zero. A mapping function is proposed by Henrick et al. [5] to obtain the optimal order near critical points. Borges et al. [6] devise a new set of WENO weights that satisfies the necessary and sufficient conditions for fifth-order convergence given by Henrick et al. [5] and enhances the accuracy at critical points. A class of higher than fifth order weighted essentially non-oscillatory schemes are designed by Balsara and Shu in [7]. Wang and Chen [8] propose optimized WENO schemes for linear waves with discontinuity. Martin et al. [9] suggest a symmetric WENO method

\footnotetext{
* Corresponding author. Tel.: +1 305284 3328; fax: +1 3052842580.

E-mail addresses: yqshen@imech.ac.cn (Y. Shen), gzha@miami.edu (G. Zha).
}

by means of a new candidate stencil, which is 2 rth-order accurate and symmetric, and less dissipative than Jiang and Shu's scheme.

The above mentioned WENO schemes are constructed to have $(2 r-1)$ th or $2 r$ th [9] order of accuracy in the smooth regions directly from $r$ th order ENO schemes. For a solution containing discontinuities, these methods can not obtain the optimal accuracy near the discontinuity points. Shen et al. [10] indicate that the smoothness indicator $I S_{k}$ of Jiang and Shu's WENO scheme does not satisfy the condition $\beta_{k}=D\left(1+O\left(\Delta x^{2}\right)\right)$ at a critical point $\left(f_{i}^{\prime}=0\right)$, and propose a step-by-step reconstruction to avoid the strict condition.

In this article, the analysis of the fifth-order WENO (WENO-5) scheme indicates that, at a transition point from smooth region to a discontinuity point or vice versa, the accuracy order of fifth order WENO scheme is decreased. Two fourth order fluxes are suggested and combined with the higher order smoothness indicators to overcome this drawback. Numerical examples show that this new method is more accurate and achieves higher resolution near discontinuities.

\section{Weighted essentially non-oscillatory schemes}

For the hyperbolic conservation law in the form

$\frac{\partial u}{\partial t}+\frac{\partial f}{\partial x}=0$

the semi-discretization form can be written as

$\frac{d u_{i}(t)}{d t}=-\frac{1}{\Delta x}\left(h_{i+\frac{1}{2}}-h_{i-\frac{1}{2}}\right)$ 
The flux $h_{i+\frac{1}{2}}$ of the classical fifth-order WENO scheme [2,6] is built through the convex combination of interpolated values $\hat{f}^{k}\left(x_{i+\frac{1}{2}}\right)(k=0,1,2)$, in which $\hat{f}^{k}(x)$ is the third degree interpolation polynomial on stencil $S_{k}^{3}=\left(x_{i+k-2}, x_{i+k-1}, x_{i+k}\right)$,

$h_{i+\frac{1}{2}}=\sum_{k=0}^{2} \omega_{k} \hat{f}^{k}\left(x_{i+\frac{1}{2}}\right)$

where

$\hat{f}^{k}\left(x_{i+\frac{1}{2}}\right)=\hat{f}_{i+\frac{1}{2}}^{k}=\sum_{j=0}^{2} c_{k j} f_{i-k+j}, \quad i=0, \cdots, N$

The weights $\omega_{k}$ are defined as

$\omega_{k}=\frac{\alpha_{k}}{\sum_{l=0}^{2} \alpha_{l}}, \quad \alpha_{k}=\frac{d_{k}}{\left(\beta_{k}+\varepsilon\right)^{p}}$

The smoothness indicators $\beta_{k}$ are given by [2]

$\beta_{k}=\sum_{l=1}^{2} \Delta x^{2 l-1} \int_{x_{i-\frac{1}{2}}}^{x_{i+\frac{1}{2}}}\left(\frac{d^{l}}{d x^{l}} \hat{f}^{k}(x)\right)^{2} d x$

For $r=3$, Eq. (6) gives

$\left\{\begin{array}{l}\beta_{0}=\frac{13}{12}\left(f_{i-2}-2 f_{i-1}+f_{i}\right)^{2}+\frac{1}{4}\left(f_{i-2}-4 f_{i-1}+3 f_{i}\right)^{2} \\ \beta_{1}=\frac{13}{12}\left(f_{i-1}-2 f_{i}+f_{i+1}\right)^{2}+\frac{1}{4}\left(f_{i-1}-f_{i+1}\right)^{2} \\ \beta_{2}=\frac{13}{12}\left(f_{i}-2 f_{i+1}+f_{i+2}\right)^{2}+\frac{1}{4}\left(3 f_{i}-4 f_{i+1}+f_{i+2}\right)^{2}\end{array}\right.$

Henrick et al. [5] show that if $\beta_{k}$ satisfy $\beta_{k}=D\left(1+O\left(\Delta x^{s}\right)\right)$, the weights $\omega_{k}$ then satisfy $\omega_{k}=d_{k}+O\left(\Delta x^{s}\right)$, where $D$ is some non-zero quantity independent of $k$. The necessary and sufficient conditions for fifth-order convergence in (2) are given as [5]:

$\sum_{k=0}^{2} A_{k}\left(\omega^{+}-\omega^{-}\right)=O\left(\Delta x^{3}\right)$

$\omega_{k}^{ \pm}-d_{k}=O\left(\Delta x^{2}\right)$

A sufficient condition for fifth-order of convergence is given by Borges et al. in [6]:

$\omega_{k}^{ \pm}-d_{k}=O\left(\Delta x^{3}\right)$

If $f_{i}^{\prime}=0$, Eq. (7) gives $\beta_{k}=D(1+O(\Delta x))$ and $\omega_{k}=d_{k}+O(\Delta x)$, which degrades the convergence accuracy of the scheme. Shen et al. [10] suggest a step-by-step reconstruction method, in which two fourth order weighted fluxes obtained from 3rd ENO fluxes are used to construct fifth order WENO scheme. Henrick et al. [5] propose a mapping function to increase the approximation of $\omega_{k}$ to the ideal weights $d_{k}$.

Borges et al. [6] introduce the absolute difference between $\beta_{0}$ and $\beta_{2}$ to devise a new set of WENO weights that satisfy the necessary and sufficient conditions for fifth-order convergence. The smoothness indicators $\beta_{k}^{z}$ defined by Borges et al. [6] are

$\beta_{k}^{z}=\frac{\beta_{k}+\varepsilon}{\beta_{k}+\tau_{5}+\varepsilon}, \quad k=0,1,2$

and the WENO weights $\omega_{k}^{z}$ of Borges et al. [6] are

$\omega_{k}^{z}=\frac{\alpha_{k}^{z}}{\sum_{l=0}^{2} \alpha_{l}^{z}}, \quad \alpha_{k}^{z}=\frac{d_{k}}{\beta_{k}^{z}}=d_{k}\left(1+\left(\frac{\tau_{5}}{\beta_{k}+\varepsilon}\right)^{q}\right), \quad k=0,1,2$

where

$\tau_{5}=\left|\beta_{0}-\beta_{2}\right|$

The coefficients $c_{k j}$ and $d_{k}$ are listed in Table 1 . The parameter $\varepsilon$ is used to avoid the division by zero $\left(\varepsilon=10^{-6}\right.$ is used in [2] and $\varepsilon=10^{-40}$ is used in [6]), $p$ and $q$ are chosen to increase the difference of scales of distinct weights at non-smooth parts of the solution. As pointed out by Borges et al. [6], for a smooth function, increasing the value of $q$ in Eq. (12) decreases the correction of the WENO-Z weights to the ideal weights $d_{k}$, making the scheme closer to the optimal central scheme. On the other hand, increasing $q$ also decreases the relative importance of the discontinuous substencil and makes the scheme more dissipative.

If $f_{i}^{\prime} \neq 0$, Eq. (12) with $q=1$ gives $\omega_{k}-d_{k}=O\left(\Delta x^{3}\right)$; if $f_{i}^{\prime}=0$, (12) with $q=2$ gives $\omega_{k}-d_{k}=O\left(\Delta x^{2}\right)$. The numerical example of Borges et al. [6] shows that, at the first-order critical point $\left(f_{i}^{\prime}=0\right)$, with $\varepsilon=10^{-40}$, WENO-JS scheme has third-order accuracy, and WENO-Z scheme with $q=1$ and $q=2$ has fourth- and fifth-order accuracy, respectively.

Fifth-order WENO schemes can capture shock waves and have fifth-order accuracy in smooth regions. However, because a WENO scheme is constructed directly from rth-order interpolation to achieve $(2 r-1)$ th-order, the accuracy is reduced at the transition point from smooth region to discontinuous point and vice versa. In order to illustrate this problem, Fig. 1 is used as an example.

At point $(i-1)$, the stencil $S_{(i-1)-1 / 2}^{5}$ is

$S_{(i-1)^{5}-1 / 2}=\left\{x_{i-4}, x_{i-3}, x_{i-2}, x_{i-1}, x_{i}\right\}$

and it is a smooth stencil, $h_{(i-1)-1 / 2}$ is obtained by the process of WENO-Z or WENO-JS as a fifth-order flux.

However, for

$S_{(i-1)+1 / 2}^{5}=\left\{x_{i-3}, x_{i-2}, x_{i-1}, x_{i}, x_{i+1}\right\}$

there is a discontinuity at stencil $S_{2}^{3}=\left\{x_{i-1}, x_{i}, x_{i+1}\right\}$, so

$\beta_{2} \gg \beta_{0}, \beta_{1}$

no matter whether WENO-Z or WENO-JS is used. To calculate the flux $h_{(i-1)+1 / 2}$ from either Eq. (5) or (12), it is easy to find

$\omega_{0} \rightarrow \frac{1}{7}, \omega_{1} \rightarrow \frac{6}{7}, \omega_{2} \rightarrow 0$

The situation at point $(i+3)$ is similar to at the point $i-1 . S_{(i+3)-1 / 2}^{5}$ contains a discontinuity at stencil $S_{0}^{3}=\left\{x_{i}, x_{i+1}, x_{i+2}\right\}$, whereas $S_{(i+3)+1 / 2}^{5}=\left\{x_{i+1}, x_{i+2}, x_{i+3}, x_{i+4}, x_{i+5}\right\}$ is a smooth stencil. For the flux $h_{(i+3)-1 / 2}$,

$\omega_{0} \rightarrow 0, \omega_{1} \rightarrow \frac{2}{3}, \omega_{2} \rightarrow \frac{1}{3}$

Let us have a look at a numerical example of a discontinuous function [6]

$u(0, x)=f(x)= \begin{cases}-\sin (\pi x)-\frac{1}{2} x^{3}, & -1<x \leqslant 0, \\ -\sin (\pi x)-\frac{1}{2} x^{3}+1, & 0<x \leqslant 1,\end{cases}$

consisting of a piecewise Sine function with a jump discontinuity at $x_{i}=0$. The weights calculated by WENO-Z scheme (Eq. (12)) is shown in Fig. 2, it demonstrates the accuracy degrading problem. For the flux $h_{(i-1)+1 / 2}, \omega_{0} \approx \frac{1}{7}$ (point $\mathrm{A}$ ), $\omega_{1} \approx \frac{6}{7}$ (point B). For $h_{(i+3)-1 / 2}, \omega_{1} \approx \frac{2}{3}$ (point D), $\omega_{1} \approx \frac{1}{3}$ (point C).

Under the condition of $\Delta x \rightarrow 0$, there are

$h_{(i-1)-\frac{1}{2}}=\frac{1}{30} f_{i-4}-\frac{13}{60} f_{i-3}+\frac{47}{60} f_{i-2}+\frac{9}{20} f_{i-1}-\frac{1}{20} f_{i}$

Table 1

Coefficients $c_{k j}$ and $d_{k}$.

\begin{tabular}{lllll}
\hline$c_{k j}$ & & & & $d_{k}$ \\
\hline $\mathrm{k}$ & $j=0$ & $j=1$ & $j=2$ & \\
\hline 0 & $1 / 3$ & $-7 / 6$ & $11 / 6$ & $1 / 10$ \\
1 & $-1 / 6$ & $5 / 6$ & $1 / 3$ & $6 / 10$ \\
2 & $1 / 3$ & $5 / 6$ & $-1 / 6$ & $3 / 10$ \\
\hline
\end{tabular}




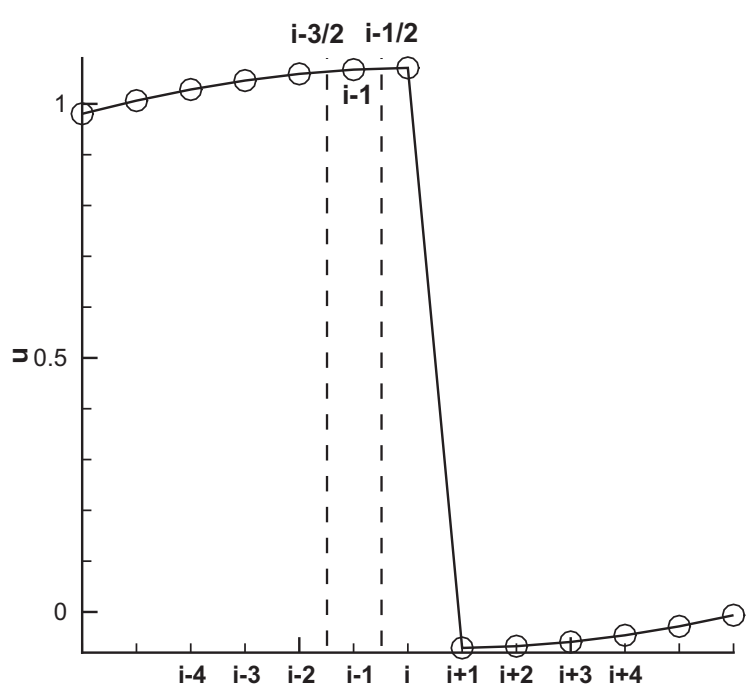

Fig. 1. The sketch of transition point.

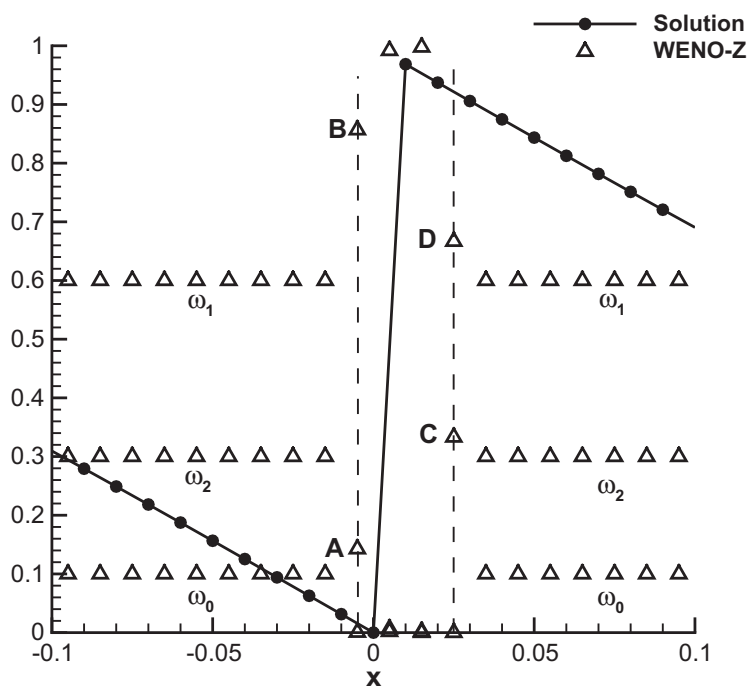

Fig. 2. The distribution of weights of WENO-Z scheme.

and

$h_{(i-1)+\frac{1}{2}}=\frac{1}{20} f_{i-3}-\frac{13}{42} f_{i-2}+\frac{41}{42} f_{i-1}+\frac{2}{7} f_{i}$

Applying Taylor series expansion, we obtain

$\frac{1}{\Delta x}\left(h_{(i-1)+\frac{1}{2}}-h_{(i-1)-\frac{1}{2}}\right)=f_{i-1}^{\prime}+O\left(\Delta x^{3}\right)$

The accuracy at the downstream point $(i+3)$ can be analyzed similarly.

That is, at the points (continuous point) immediately upstream or downstream of a discontinuity, all the current fifth-order WENO schemes only give third-order accuracy.

\section{The new method}

In this section, a new method is proposed to overcome the drawback of the fifth order WENO schemes mentioned above. The method combines the idea of the step-by-step construction of a higher order WENO scheme [10] and the properties of $\tau_{5}$ intro- duced by Borges et al. [6]. For completeness, two important properties of $\tau_{5}$ are listed here:

(1) If the stencil $S^{5}$ does not contain discontinuities, then $\tau_{5} \ll \beta_{k}$ for $k=0,1,2$;

(2) if the solution is continuous at some of the stencil $S_{i}^{3}$, but discontinuous in the whole stencil $S^{5}$, then $\beta_{i} \ll \tau_{5}$.

The new method can be described using the sketch of Fig. 3 . First, the stencils $S_{0}^{4}$ and $S_{1}^{4}$ are defined as.

$\left\{\begin{array}{l}S_{0}^{4}=S_{0}^{3} \cup S_{1}^{3}=\left\{x_{i-2}, x_{i-1}, x_{i}, x_{i+1}\right\} \\ S_{1}^{4}=S_{1}^{3} \cup S_{2}^{3}=\left\{x_{i-1}, x_{i}, x_{i+1}, x_{i+2}\right\}\end{array}\right.$

and $\tau_{4}^{0}$ and $\tau_{4}^{1}$ are defined as,

$\left\{\begin{array}{l}\tau_{4}^{0}=\left|\beta_{0}-\beta_{1}\right| \\ \tau_{4}^{1}=\left|\beta_{1}-\beta_{2}\right|\end{array}\right.$

Here, $\tau_{4}^{0}$ and $\tau_{4}^{1}$ have the same property (2) as $\tau_{5}$, i.e., if the solution is continuous at some of the stencil $S_{l+i}^{3}$, but discontinuous in the whole $S_{l}^{4}$, then $\beta_{l+i} \ll \tau_{4}^{l}$.

Next, we will analyze the relationship between $\tau_{4}^{l}$ and $\beta_{k}$ with the case of

(1) the solution is smooth on stencil $S_{0}^{4}$;

(2) the solution is discontinuous on $S_{1}^{4}$, i.e., the discontinuity is on $\left(x_{i+1}, x_{i+2}\right)$.

With condition (1), there are Taylor expansions of $\beta_{0}$ and $\beta_{1}$ as following

$\left\{\begin{array}{l}\beta_{0}=f_{i}^{\prime 2} \Delta x^{2}+\left(\frac{13}{12} f_{i}^{\prime \prime 2}-\frac{2}{3} f_{i}^{\prime} f_{i}^{\prime \prime \prime}\right) \Delta x^{4}-\left(\frac{13}{6} f_{i}^{\prime \prime} f_{i}^{\prime \prime \prime}-\frac{1}{2} f_{i}^{\prime} f_{i}^{(4)}\right) \Delta x^{5}+\left(\frac{43}{36} f_{i}^{\prime \prime \prime 2}+\frac{91}{72} f_{i}^{\prime \prime} f_{i}^{(4)}-\frac{7}{30} f_{i}^{\prime} f_{i}^{(5)}\right) \Delta x^{6}+O\left(\Delta x^{7}\right) \\ \beta_{1}=f_{i}^{\prime 2} \Delta x^{2}+\left(\frac{13}{12} f_{i}^{\prime \prime 2}+\frac{1}{3} f_{i}^{\prime} f_{i}^{\prime \prime \prime}\right) \Delta x^{4}+\left(\frac{1}{36} f_{i}^{\prime \prime 2}+\frac{13}{72} f_{i}^{\prime \prime \prime} f_{i}^{(4)}+\frac{1}{120} f_{i}^{\prime} f_{i}^{(5)}\right) \Delta x^{6}+O\left(\Delta x^{8}\right)\end{array}\right.$

Hence, if $\left(f_{i}^{\prime} \neq 0\right)$ or $\left(f_{i}^{\prime}=0\right.$ and $\left.f_{i}^{\prime} \neq 0\right)$, there is

$\tau_{4}^{0}=\left|\beta_{0}-\beta_{1}\right| \leqslant \min \left(\beta_{0}, \beta_{1}\right)$

With condition (2), there are

$\beta_{2} \gg \beta_{1}, \quad \tau_{4}^{1}=\left|\beta_{1}-\beta_{2}\right| \gg \beta_{1}$

Hence, under the conditions (1) and (2), and if $\left(f_{i}^{\prime} \neq 0\right)$ or $\left(f_{i}^{\prime}=0\right.$ and $\left.f_{i}^{\prime} \neq 0\right)$, there is

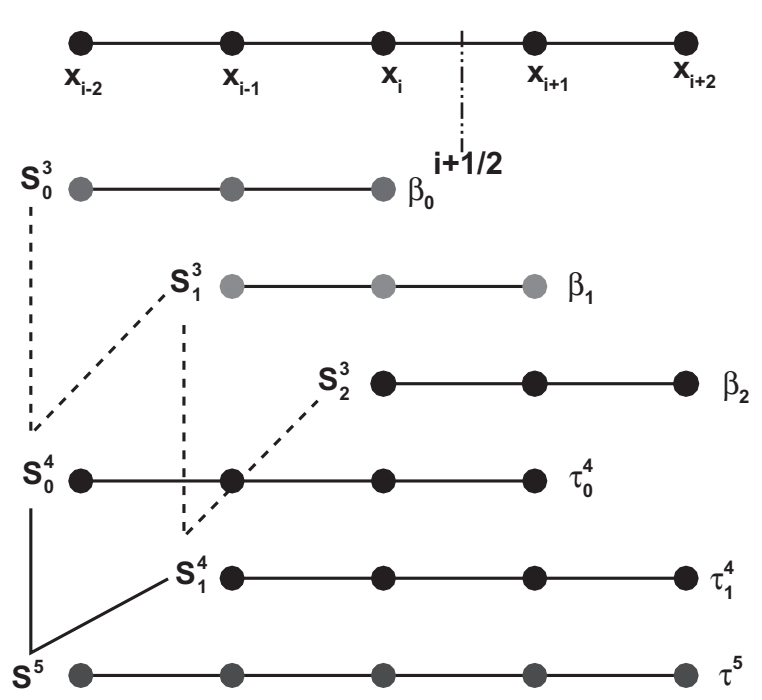

Fig. 3. The sketch of reconstruction process. 
$\left\{\begin{array}{l}\tau_{4}^{0} \leqslant \min \left(\beta_{0}, \beta_{1}, \beta_{2}\right) \\ \tau_{4}^{1}>\min \left(\beta_{0}, \beta_{1}, \beta_{2}\right)\end{array}\right.$

The same conclusion can be drawn for the case with

(1) the solution is smooth on stencil $S_{1}^{4}$;

(2) the solution is discontinuous on $S_{0}^{4}$, i.e., the discontinuity is on $\left(x_{i-2}, x_{i-1}\right)$.

Hence, the new method is constructed as

$h_{i+\frac{1}{2}}=\left\{\begin{array}{l}h_{0}^{4}, \text { if } \tau_{4}^{0} \leqslant \min \left(\beta_{0}, \beta_{1}, \beta_{2}\right) \text { and } \tau_{4}^{1}>\min \left(\beta_{0}, \beta_{1}, \beta_{2}\right), \\ h_{1}^{4}, \text { if } \tau_{4}^{0}>\min \left(\beta_{0}, \beta_{1}, \beta_{2}\right) \text { and } \tau_{4}^{1} \leqslant \min \left(\beta_{0}, \beta_{1}, \beta_{2}\right), \\ h^{\text {WENO-Z }, \text { otherwise }}\end{array}\right.$

where

$h_{0}^{4}=C_{0}^{4,0} \hat{f}_{i+1 / 2}^{0}+C_{1}^{4,0} \hat{f}_{i+1 / 2}^{1}, \quad h_{1}^{4}=C_{0}^{4,1} \hat{f}_{i+1 / 2}^{1}+C_{1}^{4,1} \hat{f}_{i+1 / 2}^{2}$

and

$C_{0}^{4,0}=\frac{1}{4}, C_{1}^{4,0}=\frac{3}{4} ; \quad C_{0}^{4,1}=\frac{1}{2}, C_{1}^{4,1}=\frac{1}{2}$

That is

$\left\{\begin{array}{l}h_{0}^{4}=\frac{1}{12} f_{i-2}-\frac{5}{12} f_{i-1}+\frac{13}{12} f_{i}+\frac{1}{4} f_{i+1} \\ h_{1}^{4}=-\frac{1}{12} f_{i-1}+\frac{7}{12} f_{i}+\frac{7}{12} f_{i+1}-\frac{1}{12} f_{i+2}\end{array}\right.$

For a smooth solution with three or more vanishing derivatives, the new scheme Eq. (28) switches to the fifth-order WENO-Z scheme.

Again, the point $(i-1)$ in Fig. 1 is taken as an example, $\left.S_{0}^{4}\right|_{i-1 / 2}=\left\{x_{i-3}, x_{i-2}, x_{i-1}, x_{i}\right\}$ is a smooth stencil, according to the properties of $\tau_{4}^{l}$, there is

$\tau_{4}^{0}<\min \left(\beta_{0}, \beta_{1}, \beta_{2}\right)$ and $\tau_{4}^{1} \gg \min \left(\beta_{0}, \beta_{1}, \beta_{2}\right)$

SO

Table 2

Results and errors.

\begin{tabular}{|c|c|c|c|c|c|c|}
\hline Point & $x_{i}$ & $f \prime\left(x_{i}\right)$ & $f \prime_{i}($ WENO-Z) & $f \prime_{i}($ present $)$ & error (WENO-Z) & error (present) \\
\hline $\mathrm{N}=40$ & $\begin{array}{l}-0.2000 \\
-0.1500 \\
-0.1000 \\
-0.0500 \\
0.0000 \\
0.0500 \\
0.1000 \\
0.1500 \\
0.2000\end{array}$ & $\begin{array}{l}-.26016 \mathrm{E}+01 \\
-.28329 \mathrm{E}+01 \\
-.30028 \mathrm{E}+01 \\
-.31067 \mathrm{E}+01 \\
-.31416 \mathrm{E}+01 \\
-.31067 \mathrm{E}+01 \\
-.30028 \mathrm{E}+01 \\
-.28329 \mathrm{E}+01 \\
-.26016 \mathrm{E}+01\end{array}$ & $\begin{array}{l}-.26016 \mathrm{E}+01 \\
-.28329 \mathrm{E}+01 \\
-.30028 \mathrm{E}+01 \\
-.31048 \mathrm{E}+01 \\
-.31343 \mathrm{E}+01 \\
0.16883 \mathrm{E}+02 \\
-.29997 \mathrm{E}+01 \\
-.28346 \mathrm{E}+01 \\
-.26016 \mathrm{E}+01\end{array}$ & $\begin{array}{l}-.26016 \mathrm{E}+01 \\
-.28329 \mathrm{E}+01 \\
-.30028 \mathrm{E}+01 \\
-.31066 \mathrm{E}+01 \\
-.31325 \mathrm{E}+01 \\
0.16883 \mathrm{E}+02 \\
-.30012 \mathrm{E}+01 \\
-.28331 \mathrm{E}+01 \\
-.26016 \mathrm{E}+01\end{array}$ & $\begin{array}{l}0.31953 \mathrm{E}-05 \\
0.25566 \mathrm{E}-05 \\
0.18554 \mathrm{E}-05 \\
0.19045 \mathrm{E}-02 \\
0.72982 \mathrm{E}-02 \\
0.19989 \mathrm{E}+02 \\
0.31294 \mathrm{E}-02 \\
0.16706 \mathrm{E}-02 \\
0.26522 \mathrm{E}-05\end{array}$ & $\begin{array}{l}0.31953 \mathrm{E}-05 \\
0.25566 \mathrm{E}-05 \\
0.18554 \mathrm{E}-05 \\
0.95956 \mathrm{E}-04 \\
0.91067 \mathrm{E}-02 \\
0.19989 \mathrm{E}+02 \\
0.15856 \mathrm{E}-02 \\
0.12686 \mathrm{E}-03 \\
0.26522 \mathrm{E}-05\end{array}$ \\
\hline $\mathrm{N}=80$ & $\begin{array}{l}-0.1000 \\
-0.0750 \\
-0.0500 \\
-0.0250 \\
0.0000 \\
0.0250 \\
0.0500 \\
0.0750 \\
0.1000\end{array}$ & $\begin{array}{l}-.30028 \mathrm{E}+01 \\
-.30632 \mathrm{E}+01 \\
-.31067 \mathrm{E}+01 \\
-.31328 \mathrm{E}+01 \\
-.31416 \mathrm{E}+01 \\
-.31328 \mathrm{E}+01 \\
-.31067 \mathrm{E}+01 \\
-.30632 \mathrm{E}+01 \\
-.30028 \mathrm{E}+01\end{array}$ & $\begin{array}{l}-.30028 \mathrm{E}+01 \\
-.30632 \mathrm{E}+01 \\
-.31067 \mathrm{E}+01 \\
-.31323 \mathrm{E}+01 \\
-.31436 \mathrm{E}+01 \\
0.36868 \mathrm{E}+02 \\
-.31052 \mathrm{E}+01 \\
-.30637 \mathrm{E}+01 \\
-.30028 \mathrm{E}+01\end{array}$ & $\begin{array}{l}-.30028 \mathrm{E}+01 \\
-.30632 \mathrm{E}+01 \\
-.31067 \mathrm{E}+01 \\
-.31328 \mathrm{E}+01 \\
-.31431 \mathrm{E}+01 \\
0.36868 \mathrm{E}+02 \\
-.31057 \mathrm{E}+01 \\
-.30632 \mathrm{E}+01 \\
-.30028 \mathrm{E}+01\end{array}$ & $\begin{array}{l}0.53282 \mathrm{E}-07 \\
0.41587 \mathrm{E}-07 \\
0.29636 \mathrm{E}-07 \\
0.52653 \mathrm{E}-03 \\
0.20075 \mathrm{E}-02 \\
0.40000 \mathrm{E}+02 \\
0.14201 \mathrm{E}-02 \\
0.43659 \mathrm{E}-03 \\
0.43276 \mathrm{E}-07\end{array}$ & $\begin{array}{l}0.53282 \mathrm{E}-07 \\
0.41587 \mathrm{E}-07 \\
0.29636 \mathrm{E}-07 \\
0.59822 \mathrm{E}-05 \\
0.14870 \mathrm{E}-02 \\
0.40000 \mathrm{E}+02 \\
0.99143 \mathrm{E}-03 \\
0.79597 \mathrm{E}-05 \\
0.43276 \mathrm{E}-07\end{array}$ \\
\hline$N=160$ & $\begin{array}{l}-0.0500 \\
-0.0375 \\
-0.0250 \\
-0.0125 \\
0.0000 \\
0.0125 \\
0.0250 \\
0.0375 \\
0.0500\end{array}$ & $\begin{array}{l}-.31067 \mathrm{E}+01 \\
-.31219 \mathrm{E}+01 \\
-.31328 \mathrm{E}+01 \\
-.31394 \mathrm{E}+01 \\
-.31416 \mathrm{E}+01 \\
-.31394 \mathrm{E}+01 \\
-.31328 \mathrm{E}+01 \\
-.31219 \mathrm{E}+01 \\
-.31067 \mathrm{E}+01\end{array}$ & $\begin{array}{l}-.31067 \mathrm{E}+01 \\
-.31219 \mathrm{E}+01 \\
-.31328 \mathrm{E}+01 \\
-.31393 \mathrm{E}+01 \\
-.31425 \mathrm{E}+01 \\
0.76861 \mathrm{E}+02 \\
-.31324 \mathrm{E}+01 \\
-.31220 \mathrm{E}+01 \\
-.31067 \mathrm{E}+01\end{array}$ & $\begin{array}{l}-.31067 \mathrm{E}+01 \\
-.31219 \mathrm{E}+01 \\
-.31328 \mathrm{E}+01 \\
-.31394 \mathrm{E}+01 \\
-.31423 \mathrm{E}+01 \\
0.76861 \mathrm{E}+02 \\
-.31325 \mathrm{E}+01 \\
-.31219 \mathrm{E}+01 \\
-.31067 \mathrm{E}+01\end{array}$ & $\begin{array}{l}0.84590 \mathrm{E}-09 \\
0.65623 \mathrm{E}-09 \\
0.46551 \mathrm{E}-09 \\
0.14246 \mathrm{E}-03 \\
0.89829 \mathrm{E}-03 \\
0.80000 \mathrm{E}+02 \\
0.42399 \mathrm{E}-03 \\
0.11444 \mathrm{E}-03 \\
0.68340 \mathrm{E}-09\end{array}$ & $\begin{array}{l}0.84590 \mathrm{E}-09 \\
0.65623 \mathrm{E}-09 \\
0.46551 \mathrm{E}-09 \\
0.37364 \mathrm{E}-06 \\
0.75621 \mathrm{E}-03 \\
0.80000 \mathrm{E}+02 \\
0.31005 \mathrm{E}-03 \\
0.49793 \mathrm{E}-06 \\
0.68340 \mathrm{E}-09\end{array}$ \\
\hline
\end{tabular}

$h_{(i-1)+1 / 2}=h_{0}^{4}=\frac{1}{12} f_{i-3}-\frac{5}{12} f_{i-2}+\frac{13}{12} f_{i-1}+\frac{1}{4} f_{i}$

Meanwhile, $S_{i-3 / 2}^{5}$ is a smooth stencil, $h_{(i-1)-1 / 2}$ keeps the fifth-order flux $h_{(i-1)-1 / 2}^{\text {WENO } Z}$ (Eq. (20)). Hence, applying Taylor series expansion, there is

$\frac{1}{\Delta x}\left(h_{(i-1)+\frac{1}{2}}-h_{(i-1)-\frac{1}{2}}\right)=f_{i-1}^{\prime}+O\left(\Delta x^{4}\right)$

Compared with the accuracy of the original WENO-Z or WENO-JS scheme (Eq. (22)), the new method improves one accuracy order at the point right next to the discontinuity $(i-1)$.

Table 2 gives the comparison of values and errors of WENO-Z scheme and the present method of first-order derivative of $f(x)$ given by Eq. (19) near the discontinuity points. For this case, $x_{i}=0$ and the next point $x_{i+1}$ are the discontinuity points. At points $x_{i-1}$ and $x_{i+3}$, the present method is clearly more accurate than WENO-Z scheme.

It should be pointed out that the transition point is actually still a smooth point. Hence theoretically, the finite difference approximation of the first order derivative can reach up to fourth order accuracy by using five smooth (continuous) points. However, the accuracy at the transition point is influenced greatly by the accuracy of its neighboring points, especially at the discontinuity points. As claimed by Engquist and Sjogreen [11], the accuracy of high order difference schemes is reduced to first order at a shock. For this reason, even a small improvement of the accuracy at the transition point is very desirable for simulation of the flows with shock wave/complex flow structures interaction.

\subsection{Numerical examples}

In this paper, the fourth order Runge-Kutta-type method [12] is used for the time marching. 
Table 3

Accuracy on $u_{t}+u_{x}=0$ with $u_{0}(x)=\sin (2 \pi x), t=1$.

\begin{tabular}{|c|c|c|c|c|c|}
\hline Scheme & $N$ & $L_{\infty}$ error & $L_{\infty}$ order & $L_{1}$ error & $L_{1}$ order \\
\hline \multirow[t]{5}{*}{ WENO-Z } & 40 & $0.315356 \mathrm{E}-03$ & -- & $0.198512 \mathrm{E}-03$ & -- \\
\hline & 80 & $0.994903 \mathrm{E}-05$ & 4.986 & $0.628679 \mathrm{E}-05$ & 4.981 \\
\hline & 160 & $0.312476 \mathrm{E}-06$ & 4.993 & $0.197921 \mathrm{E}-06$ & 4.989 \\
\hline & 320 & $0.977606 \mathrm{E}-08$ & 4.998 & $0.620618 \mathrm{E}-08$ & 4.995 \\
\hline & 640 & $0.305593 \mathrm{E}-09$ & 5.000 & $0.194260 \mathrm{E}-09$ & 4.998 \\
\hline \multirow[t]{5}{*}{ present } & 40 & $0.315356 \mathrm{E}-03$ & -- & $0.198512 \mathrm{E}-03$ & -- \\
\hline & 80 & $0.994903 \mathrm{E}-05$ & 4.986 & $0.628679 \mathrm{E}-05$ & 4.981 \\
\hline & 160 & $0.312476 \mathrm{E}-06$ & 4.993 & $0.197921 \mathrm{E}-06$ & 4.989 \\
\hline & 320 & $0.977606 \mathrm{E}-08$ & 4.998 & $0.620618 \mathrm{E}-08$ & 4.995 \\
\hline & 640 & $0.305593 \mathrm{E}-09$ & 5.000 & $0.194260 \mathrm{E}-09$ & 4.998 \\
\hline
\end{tabular}

\subsubsection{Linear transport equation}

The linear transport equation is used to test the accuracy of WENO schemes.

$\frac{\partial u}{\partial t}+\frac{\partial u}{\partial x}=0,-1<x<1$

$u(x, 0)=u_{0}(x)$, periodic

(1) We start with the advection of a smooth profile

$$
u_{0}(x)=\sin (2 \pi x)
$$

Table 3 gives the errors and accuracy order. It can be seen that for this smooth solution, the present scheme obtains the same results and accuracy order as the WENO-Z scheme.

(2) A solution with an initial discontinuity [6] is calculated

$$
u(0, x)=f(x)= \begin{cases}-\sin (\pi x)-\frac{1}{2} x^{3}, & -1<x \leqslant 0, \\ -\sin (\pi x)-\frac{1}{2} x^{3}+1, & 0<x \leqslant 1,\end{cases}
$$

Figs. 4 and 5 show the numerical solutions and errors at $t=10$. Near the discontinuity $(x=0)$, the range of error is about $\left(10^{-2}, 1\right)$, and the present method improves the accuracy obviously. While in the other smooth regions, the errors are relatively small, and the difference of two schemes is also negligible.

(3) A more complex initial solution is tested

$$
u_{0}(x)= \begin{cases}-x \sin \left(3 \pi x^{2} / 2\right), & -1 \leqslant x<-1 / 3 \\ |\sin (2 \pi x)|, & -1 / 3 \leqslant x \leq 1 / 3 \\ 2 x-1-\sin (3 \pi x) / 6, & \text { otherwise }\end{cases}
$$

Figs. 6 and 5 show the numerical solutions and errors at $t=6$. Again, it can be seen that the present method is more accurate. (see Fig. 7).

(4) Finally, a more challenging test case that contains a smooth combination of Gaussians, a square wave, a sharp triangle wave, and a half ellipse is calculated.

$$
u_{0}(x)= \begin{cases}\frac{1}{6}(G(x, \beta, z-\delta)+G(x, \beta, z+\delta)+4 G(x, \beta, z)), & -0.8 \leqslant x \leqslant-0.6, \\ 1, & -0.4 \leqslant x \leqslant-0.2, \\ 1-|10(x-0.1)|, & 0 \leqslant x \leqslant 0.2, \\ \frac{1}{6}(F(x, \alpha, \alpha-\delta)+F(x, \alpha, \alpha+\delta)+4 F(x, \alpha, a)), & 0.4 \leqslant x \leqslant 0.6, \\ 0, & \text { otherwise }\end{cases}
$$

As in Ref. [2], the constants for this case are taken as $a=0.5, z=-0.7, \delta=0.005, \alpha=10$, and $\beta=\log 2 / 36 \delta^{2}$.

The results at $t=8$ with 200 grid points are shown in Figs. 8 and 9. From the zoomed plots in Fig. 10, it can be seen that the present method improves the accuracy not only near the discontinuities, but also for the peak of the half ellipse wave.

\subsubsection{Nonlinear transport equation}

The nonlinear transport equation can be written as

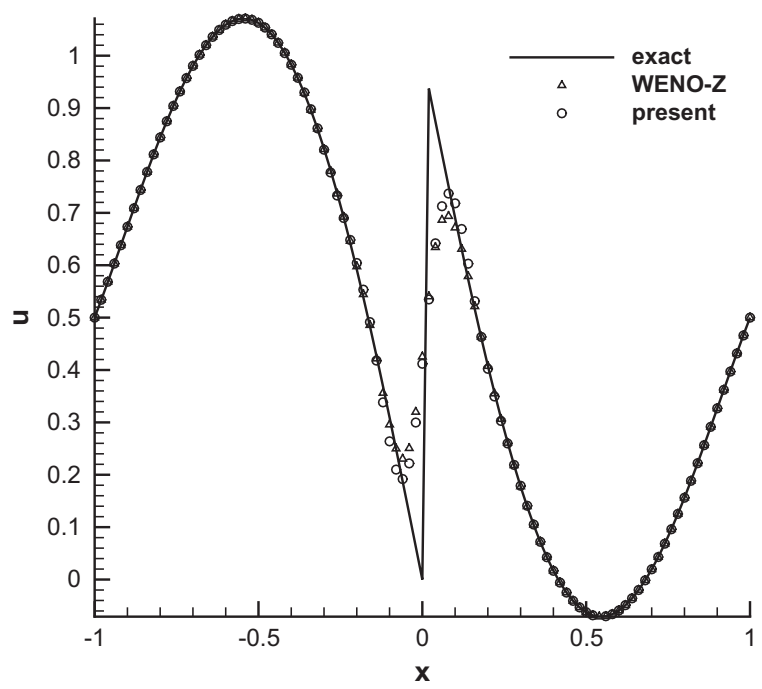

Fig. 4. Numerical results of linear transport equation, initial condition (32). $t=10$.

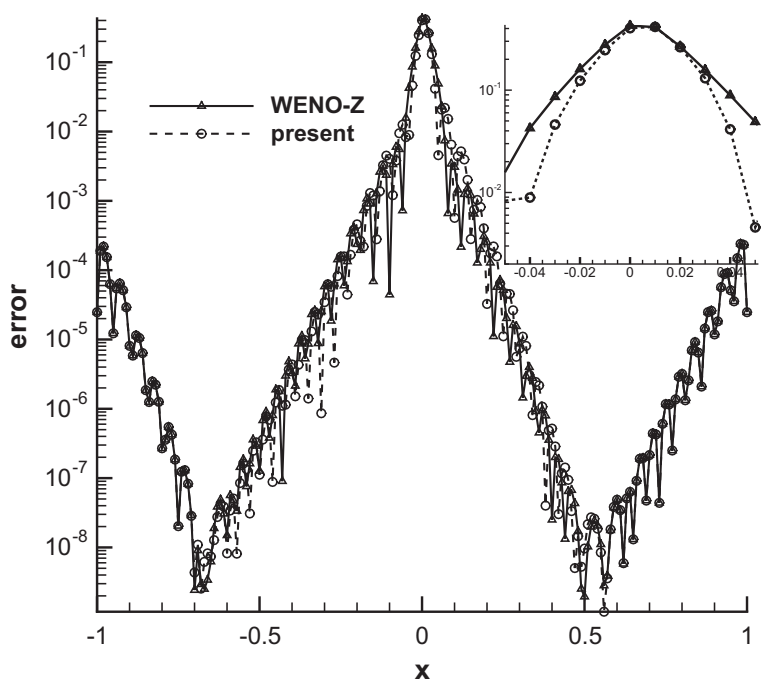

Fig. 5. Absolute pointwise error, initial condition (32). $t=10$.

$\frac{\partial u}{\partial t}+u \frac{\partial u}{\partial x}=0,0 \leqslant x \leqslant 2 \pi$

with initial and boundary conditions

$u_{0}(x)=0.3+0.7 \sin (x), 0 \leqslant x \leqslant 2 \pi$, periodic 


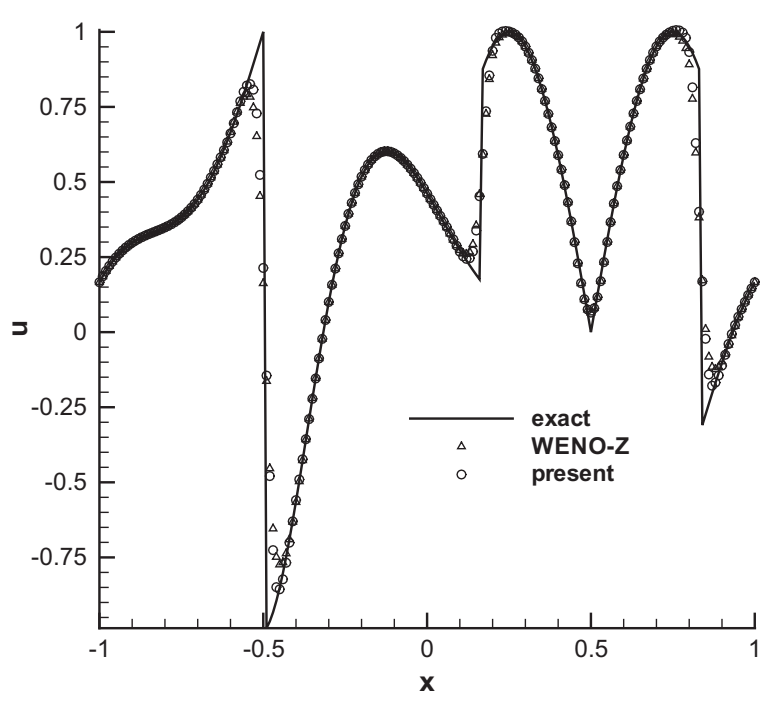

Fig. 6. Numerical results of linear transport equation, initial condition (33). $t=6$.

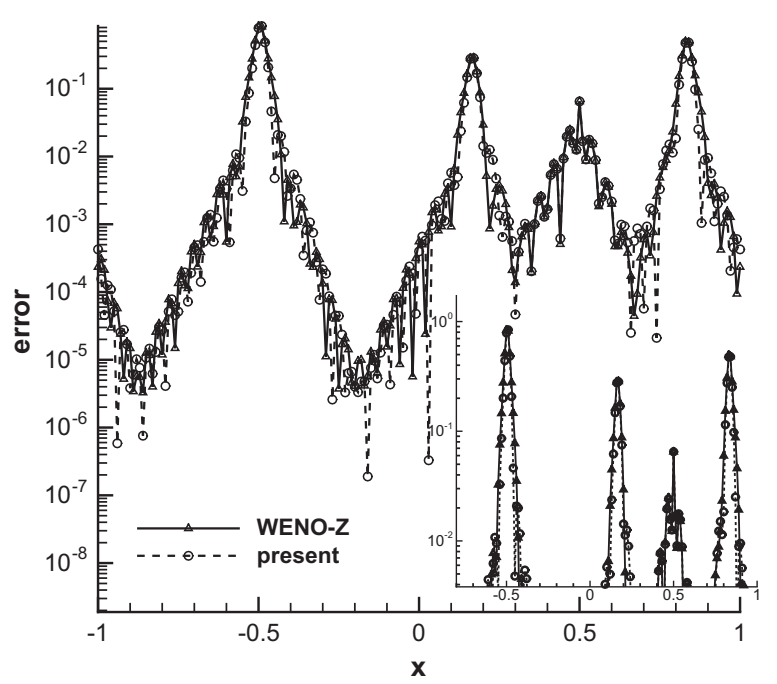

Fig. 7. Absolute pointwise error, initial condition (33). $t=6$.

The Lax-Friedrichs splitting method is used, in which $f^{ \pm}=\frac{1}{2}(f(u) \pm a u), f(u)=\frac{1}{2} u^{2}$, and $a=\max _{u}\left|f^{\prime}(u)\right|$. Fig. 11 shows the results at $t=2$ with grid number of $N=80$. It can be seen that, near the shock, the solution calculated by the present scheme is closer to the discontinuous points than those of WENO-Z scheme.

\subsection{3. $1 D$ Shock Wave Tube, Sod Problem}

To examine the new scheme for nonlinear equations, the onedimensional Euler equations are solved for the 1D shock tube problem.

1D Euler equations:

$\frac{\partial \mathbf{U}}{\partial t}+\frac{\partial \mathbf{F}}{\partial x}=0$

where

$\mathbf{U}=\left[\begin{array}{l}\rho \\ \rho u \\ \rho e\end{array}\right] \mathbf{F}=\left[\begin{array}{c}\rho u \\ \rho u^{2}+p \\ u(\rho e+p)\end{array}\right], p=(\gamma-1)\left(\rho e-\rho u^{2} / 2\right), \gamma=1.4$.

The initial condition is

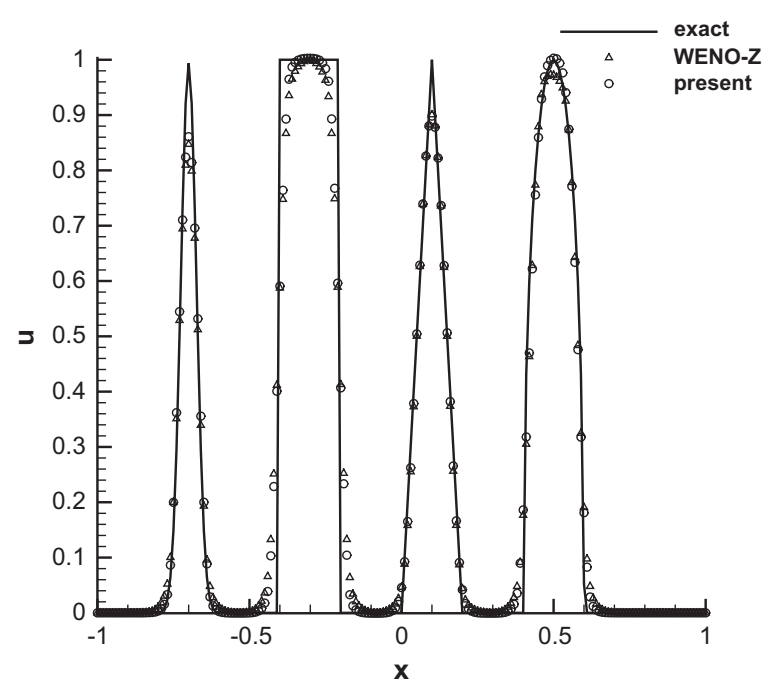

Fig. 8. Numerical results of linear transport equation, initial condition (34). $t=8$.

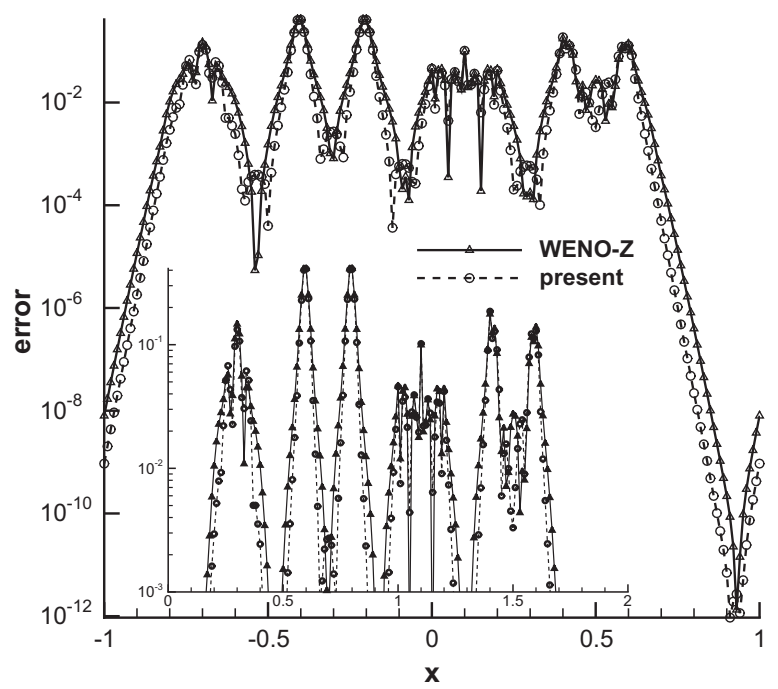

Fig. 9. Absolute pointwise error, initial condition (34). $t=8$.

$(\rho, u, p)= \begin{cases}(1.0,0.0,1.0), & x \leqslant 7.5 \\ (0.125,0.0,0.1), & x>7.5\end{cases}$

In this case, the Roe's Riemann solver is used. The grid points is $N=200$. Fig. 12 give the density distribution. Both the WENO-Z and present schemes capture the shock very well. The present scheme improves the resolution near the discontinuities.

\subsubsection{D Shock Wave Tube, Shu-Osher Problem}

This problem is governed by the one-dimensional Euler Eq. (35) with following initial condition:

$(\rho, u, p)= \begin{cases}(3.857143,2.629369,10.3333), & \text { when } x<-4 \\ (1+\varepsilon \sin (5 x), 0.0,1.0), & \text { when } x \geqslant-4\end{cases}$

where, $\varepsilon=0.2$. This case represents a Mach 3 shock wave interacting with a Sine entropy wave [4]. The results at time $t=1.8$ with mesh size of 200 are plotted in Fig. 13. The "exact" solutions are the numerical solutions of the original WENO-5 scheme with grid points of $N=8000$. For this case, it can be seen that the present WENO scheme resolves the profile better than the WENO-Z scheme. 

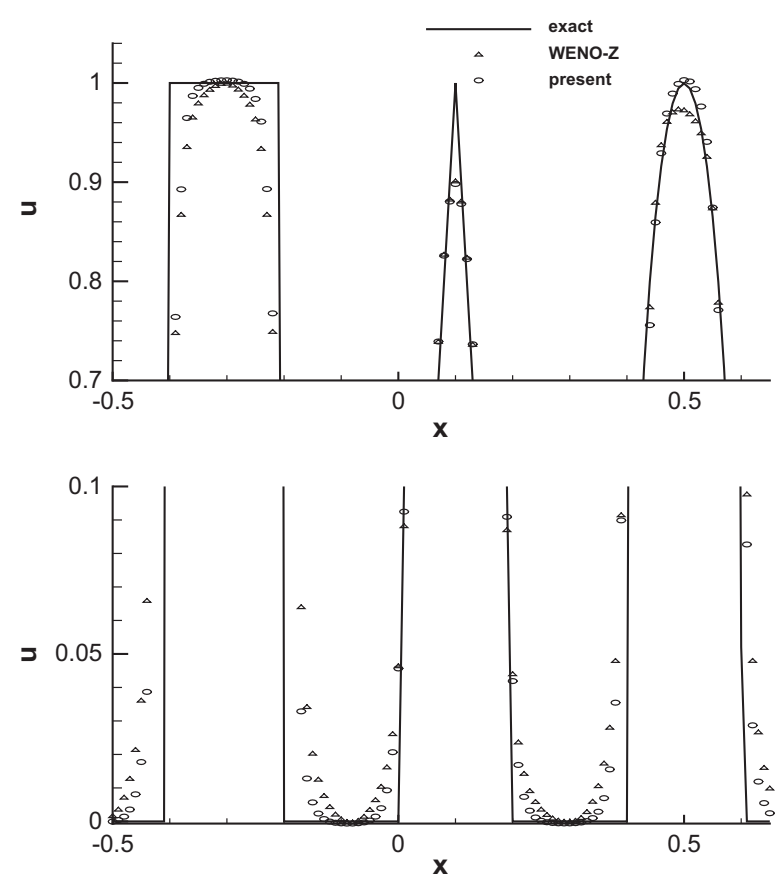

Fig. 10. Locally enlarged plot of Fig. 8.

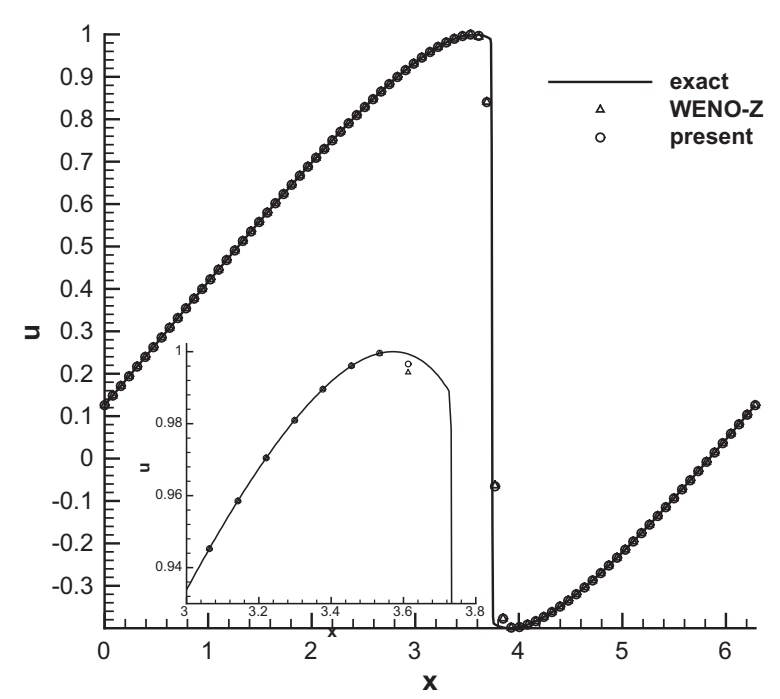

Fig. 11. Numerical results of nonlinear transport equation, $t=2$.

3.1.5. Two-dimensional Linear Conservation Equation with Variable Coefficients

To test the multidimensional problems, the 2D linear conservation equation with variable coefficients is solved. The governing equation is

$\frac{\partial u}{\partial t}+\frac{\partial(-y u)}{\partial x}+\frac{\partial(x u)}{\partial y}=0,-1 \leqslant x, y \leqslant 1$

and the periodic boundary conditions are used. The initial condition is chosen as the characteristic function of a circle with radius 0.5 as shown in Fig. 14. The problem represents a solid body rotation $[13,14]$. The results at $t=2$ in a $100 \times 100$ points grid are shown in Fig. 15. Note that in Fig. 15, the exact solution at $x=-0.52$ is $u(-0.52, y)=0$. It can be seen that the present scheme obtains

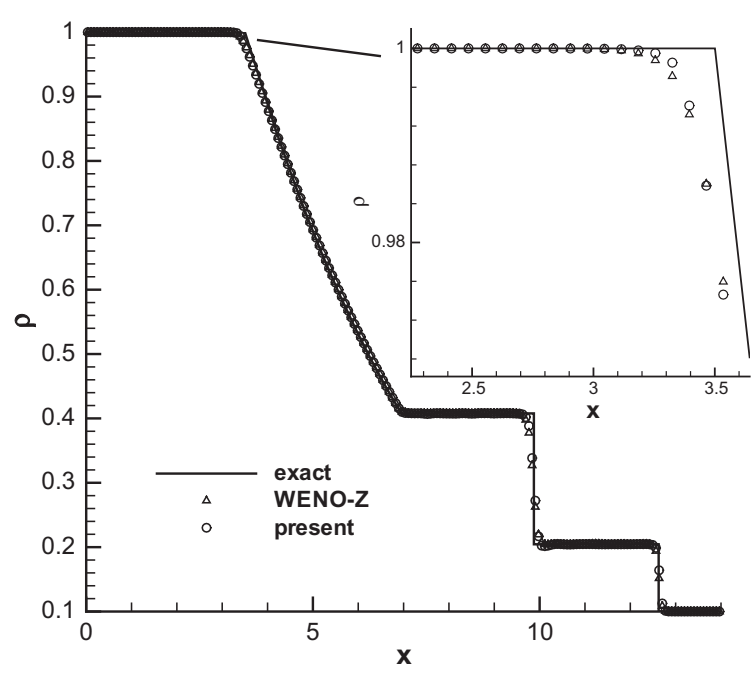

Fig. 12. Density distribution of 1D shock tube, Sod problem.

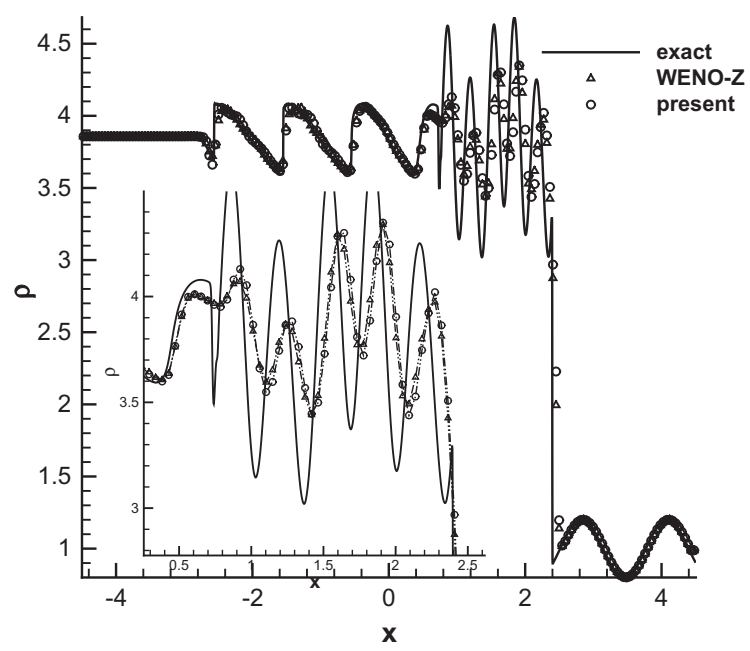

Fig. 13. Density distribution of 1D shock tube, Shu-Osher problem.

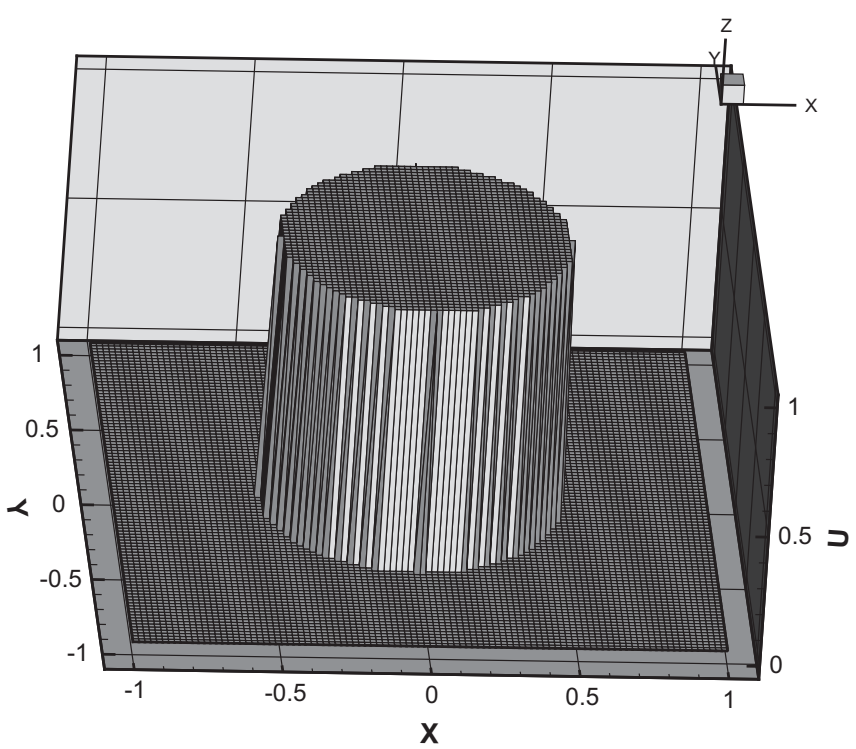

Fig. 14. The initial distribution, the $2 \mathrm{D}$ variable coefficients problem. 
more accurate solution than the WENO-Z scheme, especially at the location $x=-0.48$ and $x=-0.52$.

\subsubsection{Two-dimensional Shock Vortex Interaction}

A two-dimensional shock vortex interaction problem is solved to further demonstrate the high resolution of the present scheme. The two-dimensional Euler equations are solved for this problem:

$\frac{\partial \mathbf{U}}{\partial t}+\frac{\partial \mathbf{E}}{\partial x}+\frac{\partial \mathbf{F}}{\partial y}=0$

where

$\mathbf{U}=\left[\begin{array}{c}\rho \\ \rho u \\ \rho v \\ \rho e\end{array}\right], \mathbf{E}=\left[\begin{array}{c}\rho u \\ \rho u^{2}+p \\ \rho u v \\ u(\rho e+p)\end{array}\right], \mathbf{F}=\left[\begin{array}{c}\rho v \\ \rho u v \\ \rho v^{2}+p \\ v(\rho e+p)\end{array}\right]$,

$p=(\gamma-1)\left(\rho e-\rho\left(u^{2}+v^{2}\right) / 2\right), \gamma=1.4$.

The problem is taken from Jiang and Shu [2]. It describes the interaction between a stationary shock and a vortex. The computational domain is taken to be $[0,2] \times[0,1]$. A stationary Mach 1.1 shock is

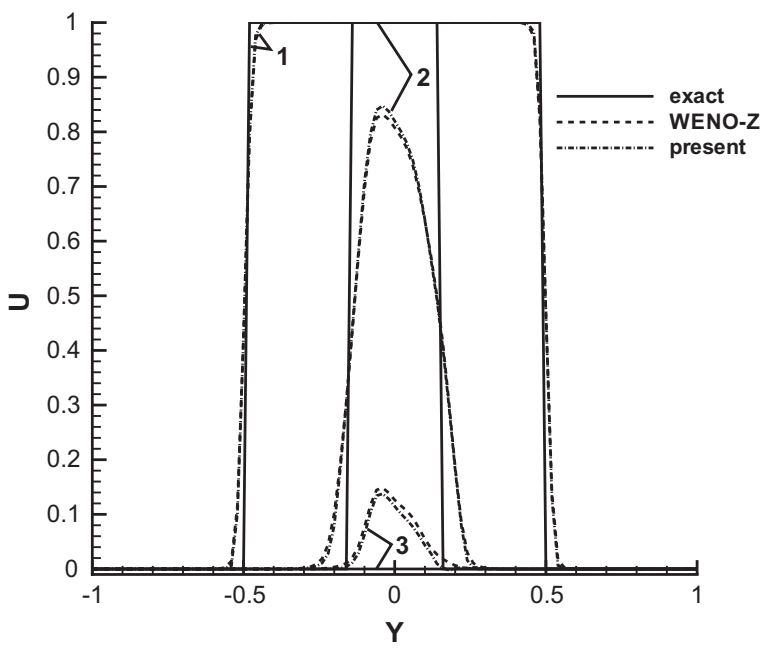

Fig. 15. Comparison of results on lines, the $2 \mathrm{D}$ variable coefficients problem. 1 : $x=-0.02 ; 2: x=-0.48$; and $3: x=-0.52$

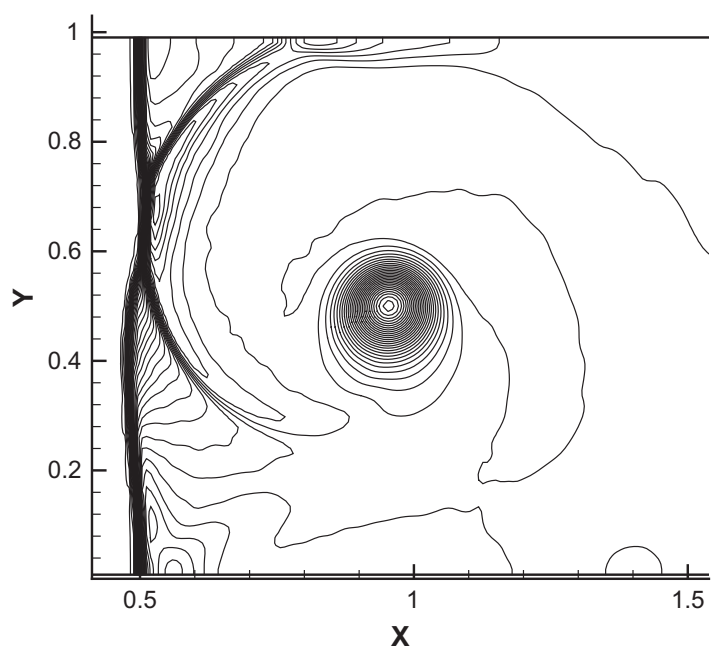

Fig. 16. The pressure contours of present scheme, the 2D shock vortex interaction flow. $t=0.60$ positioned at $x=0.5$ and normal to the $x$-axis. Its left state is $(\rho, u, v, p)=(1,1.1 \sqrt{\gamma}, 0,1)$. A small vortex is superimposed to the flow on the left of the shock and is centered at $\left(x_{c}, y_{c}\right)=(0.25,0.5)$. The vortex is described as a perturbation to the velocity $(u, v)$, temperature $(T=p / \rho)$, and entropy $\left(S=\ln \left(p / \rho^{\gamma}\right)\right)$ of the mean flow and denoted by the tilde values:

$\tilde{u}=\varepsilon \tau e^{a\left(1-\tau^{2}\right)} \sin \theta$

$\tilde{v}=-\varepsilon \tau e^{a\left(1-\tau^{2}\right)} \cos \theta$

$\widetilde{T}=-\frac{(\gamma-1) \varepsilon^{2} e^{2 a\left(1-\gamma^{2}\right)}}{4 a \gamma}$

$\widetilde{S}=0$

where $\tau=r / r_{c}$ and $r=\sqrt{\left(x-x_{c}\right)^{2}+\left(y-y_{c}\right)^{2}}, \varepsilon$ indicates the strength of the vortex, $a$ controls the decay rate of the vortex, and $r_{c}$ is the critical radius for which the vortex has the maximum strength. As in the Refs. [2,15], $\varepsilon=0.3, r_{c}=0.05$, and $a=0.204$ are adopted in this paper. The time step is taken as follows [16]:

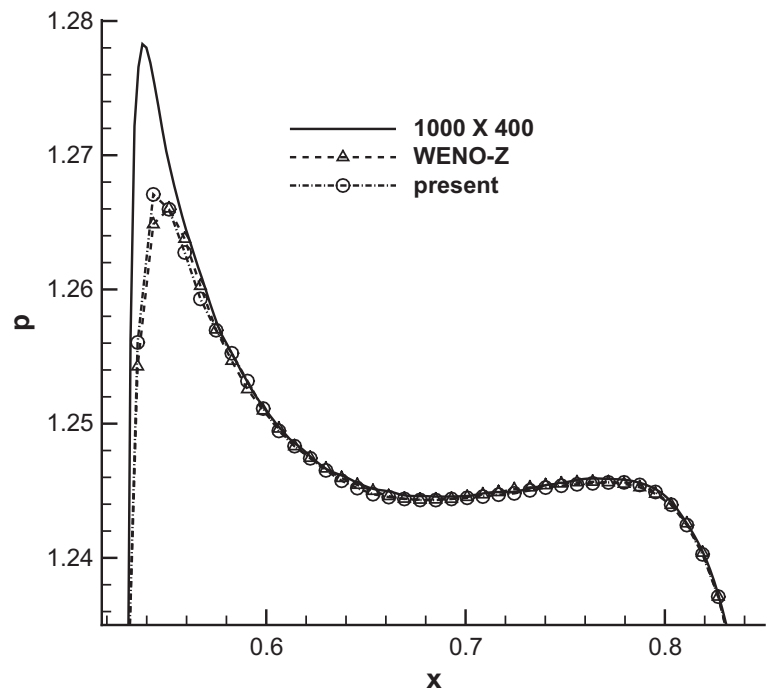

Fig. 17. Comparison of pressure at the central line downstream the shock, the 2D shock vortex interaction flow. $t=0.60$

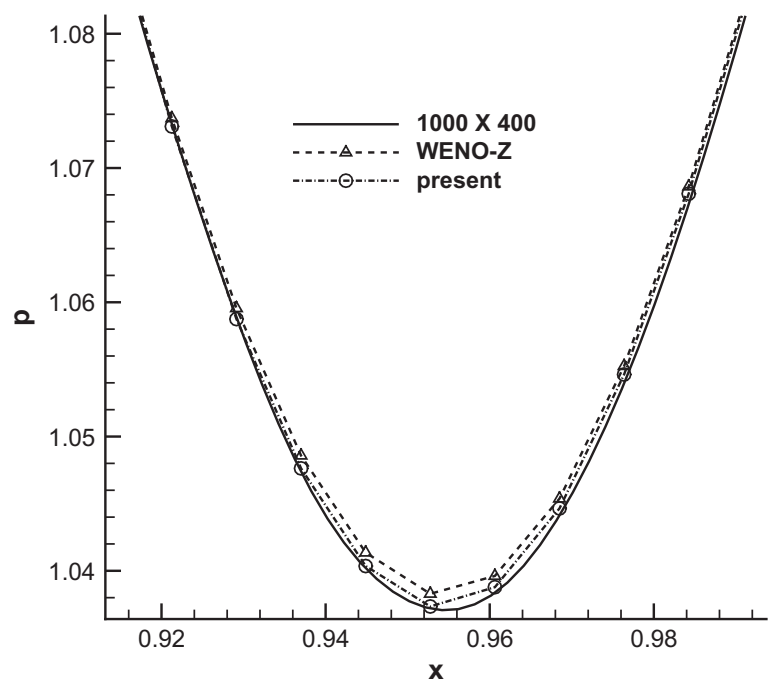

Fig. 18. Comparison of pressure at the central line cross the vortex, $t=0.60$ 


$$
\begin{aligned}
& \Delta t=\delta \frac{\Delta t_{x} \Delta t_{y}}{\Delta t_{x}+\Delta t_{y}}, \text { with } \Delta t_{x}=\frac{\Delta x}{\max _{i, j}\left(\left|u_{i, j}\right|+c_{i, j}\right)}, \\
& \Delta t_{y}=\frac{\Delta y}{\max _{i, j}\left(\left|v_{i, j}\right|+c_{i, j}\right)}
\end{aligned}
$$

where $\delta=0.5$ is the CFL number.

Fig. 16 is the pressure contours at $t=0.60$. Figs. 17 and 18 are the comparisons of the pressure between the present and the original scheme along the center line at $y=0.5$. In order to show the accuracy of the new scheme, the results obtained by the WENO-Z scheme with a refined mesh of $1001 \times 401$ is also given. With the same coarse mesh density of $251 \times 101$, the new scheme obtains more accurate results than the original scheme. Fig. 17 also shows that the new scheme has the sharper shock profile. Fig. 18 indicates that the new scheme achieves lower vortex core pressure due to lower numerical dissipation.

\section{Conclusions}

The analysis of this paper indicates that all the current WENO schemes reduce their order of accuracy at the transition points near discontinuities to third order. A new method combining the forth-order fluxes with higher order smoothness indicators is suggested and overcomes this drawback. The order of accuracy immediately next to a discontinuity is improved to fourth order, whereas the order of accuracy in smooth regions is maintained as fifth order. Numerical examples with 1D linear and nonliner Euler equations, 2D Euler equations, all show that the new scheme is more accurate to resolve the flow solutions near discontinuities.

\section{Acknowledgments}

This work is partially supported by AFOSR Grant FA9550-06-10198 monitored by Dr. Fariba Fahroo, by ARO Grant 56827-RT-ISP monitored by John Schmisseur at AFOSR and Peggy A. Lacewell at ARO, and by Miami Wind ${ }^{\mathrm{TM}}$ at University of Miami.

\section{References}

[1] Liu XD, Osher S, Chan T. Weighted essentially non-oscillatory schemes. J Comput Phys 1994;115:200-12.

[2] Jiang G-S, Shu C-W. Efficient implementation of weighted ENO schemes. J Comput Phys 1996;126:202-28.

[3] Harten A, Engquist B, Osher S, Chakravarthy S. Uniformly high order essentially non-oscillatory schemes, III. J Computat Phys 1987;71:231-303.

[4] Shu C-W, Osher O. Efficient implementation of essentially non-oscillatory shock capturing schemes, II. J Computat Phys 1989;83:32-78.

[5] Henrick AK, Aslam TD, Powers JM. Mapped weighted essentially nonoscillatory schemes: achiving optimal order near critical points. J Comput Phys 2005;208:206-27.

[6] Borges R, Carmona M, Costa B, Don WS. An improved weighted essentially non-oscillatory scheme for hyperbolic conservation laws. J Computat Phys 2008;227:3191-211.

[7] Balsara DS, Shu C-W. Monotonicity preserving weighted essentially nonoscillatory schemes with increasingly high order of accuracy. J Comput Phys 2000;160:405-52.

[8] Wang ZJ, Chen RF. Optimized weighted essentially non-oscillatory schemes for linear waves with discontinuity. J Comput Phys 2001:174:381-404.

[9] Martin MP, Taylor EM, Wu M, Weirs VG. A bandwidth-optimized WENO scheme for the direct numerical simulation of compressible turbulence. J Computat Phys 2006;220:270-89.

[10] Shen Y-Q, Wang R-Q, Liao H-Z. A fifth-order accurate weighted ENN difference scheme and its applications. J Computat Math 2001;19:531-8.

[11] Engquist B, Sjogreen B. The convergence rate of finite difference schemes in the presence of shocks. SIAM J Numer Anal 1998;35:2464-85.

[12] Shu C-W, Osher O. Efficient implementation of essentially non-oscillatory shock capturing schemes. J Computat Phys 1988;77:439-71.

[13] Cockburn B, Shu CW. Nonlinearly stable compact schemes for shock calculations. SIAM J Numer Anal 1994;31:607-27.

[14] Shen Y-Q, Yang G-W, Gao Z. High-resolution finite compact difference schemes for hyperbolic conservation laws. J Comput Phys 2006;216:114-37.

[15] Shen Y-Q, Yang G-W. Hybrid finite compact-WENO schemes for shock calculation. Int J Numer Methods Fluids 2007;53:531-60.

[16] Pirozzoli S. Conservative hybrid compact-WENO schemes for shockturbulence interaction. J Comput Phys 2002:178:81-117. 